\title{
A novel clinical multidimensional transcriptome signature predicts prognosis in bladder cancer
}

\author{
CHUN-PENG LIU ${ }^{1 *}$, JIAN-HUA ZHANG ${ }^{2 *}$, SHUAI-CHAO ZHENG ${ }^{3}$, JING LIU $^{4}$ and JIN-CHENG GUO ${ }^{5}$ \\ ${ }^{1}$ Department of Pathology, Shantou Central Hospital, Affiliated Shantou Hospital of Sun Yat-sen University, Shantou, \\ Guangdong 515041; ${ }^{2}$ Department of Blood Transfusion, Peking University People's Hospital, Beijing 100000; \\ ${ }^{3}$ State Key Laboratory for Conservation and Utilization of Bio-Resources in Yunnan, Yunnan University, \\ Kunming, Yunnan 650000; ${ }^{4}$ College of Life Sciences, Northwest University, Xi'an, Shaanxi 710069; \\ ${ }^{5}$ Department of Biochemistry and Molecular Biology, Shantou University Medical College, \\ Shantou, Guangdong 515100, P.R. China
}

Received March 15, 2018; Accepted August 13, 2018

DOI: $10.3892 /$ or.2018.6677

\begin{abstract}
A number of studies has shown that long non-coding RNAs (lncRNAs), microRNAs (miRNAs) and protein coding genes (PCGs) are involved in various pathophysiological processes and can be used as prognostic biomarkers in cancer patients. The purpose of this study was to find a multidimensional transcriptome signature to predict clinical outcomes in bladder cancer. Using Cox's proportional hazards regression analysis and the random survival forest algorithm, we mined the expression profile data of 239 bladder cancer patients derived from The Cancer Genome Atlas (TCGA) public database. A signature comprised of two PCGs (ACADS and C1QTNF9B), two lncRNAs (RP11-60L3.1 and CTD-3195I5.3) and two microRNAs (has-miR-3913-1 and has-miR-891a) with highest accuracy prediction (AUC $=0.79$ in the training dataset and 0.64 in the test dataset) was selected. The signature had an ability to
\end{abstract}

Correspondence to: Mr. Chun-Peng Liu, Department of Pathology, Shantou Central Hospital, Affiliated Shantou Hospital of Sun Yat-Sen University, 114 Waima Road, Shantou, Guangdong 515041, P.R. China

E-mail: 510130090@qq.com

Mr. Jin-Cheng Guo, Department of Biochemistry and Molecular Biology, Shantou University Medical College, 22 Xinling Road, Shantou, Guangdong 515100, P.R. China

E-mail: obgene@qq.com

*Contributed equally

Abbreviations: ROC, receiver operating characteristic; AUC, area under the ROC curve; CI, confidence interval; GO, Gene Ontology; HR, hazard ratio; KEGG, Kyoto Encyclopedia of Genes and Genomes; lncRNAs, long non-coding RNAs; OS, overall survival; BLCA, bladder urothelial carcinoma; SD, standard deviation; TCGA, The Cancer Genome Atlas

Key words: bladder cancer, protein-coding genes, long non-coding RNA, microRNA, TCGA, prognostic marker stratify patients into high- and low-risk groups with significantly different survival rates (median 16.9 vs. 54.9 months, log-rank test $\mathrm{P}<0.001)$ in the training dataset, and its performance was validated for risk stratification in the test dataset (median 18.2 vs. 58.9 months, log-rank test $\mathrm{P}=0.002$ ). Multivariable Cox regression analysis revealed that the signature was an independent prognostic factor for patients with bladder urothelial carcinoma (BLCA). A comparison of tumour node metastasis (TNM) stage and the signature indicated that the signature had better survival prediction power $\left(\mathrm{AUC}_{\text {signature }}=0.79 / 0.64\right.$ vs. $\left.\mathrm{AUC}_{\mathrm{TNM}}=0.67 / 0.60, \mathrm{P}<0.05\right)$. Functional analyses indicated that these prognostic genes from the signature may be involved in tumourigenesis-related biological processes and pathways. In conclusion, the multidimensional PCG-IncRNA-microRNA signature can be a novel prognostic marker to predict the survival of bladder cancer patients.

\section{Introduction}

Bladder cancer, prevalent in men over 65 years old, is a globally common urinary tract malignancy (1). Generally, bladder cancer refers to bladder urothelial carcinoma (BLCA), and BLCA accounts for $90 \%$ of bladder cancers. According to studies by the American Cancer Society, $96 \%$ of bladder cancer in situ patients survive 5 years or more. Hence, the prognosis of patients with bladder cancer is better than that of patients with other malignant tumours such as pancreatic carcinoma, lung cancer, hepatocellular carcinoma or oesophageal cancer. However, a high recurrence rate and tumour progression makes the prognosis of bladder cancer patients worse. Thus, the five-year survival rate of bladder cancer decreases to $5-70 \%$ (2). Currently, tumour node metastasis (TNM) classification and pathological grade are widely used clinically to plan treatment and predict prognosis. However, they are indicators at a population level, incapable of precisely predicting individual clinical outcomes (3). In clinical practice, the individual clinical outcome is considered more meaningful to guide clinical therapy and evaluate prognosis, if we know this personal information in advance, including disease recurrence risk, therapeutic response and overall survival (OS). 
Therefore, new prognostic and predictive biomarkers are urgently needed for bladder cancer patients.

Due to The Cancer Genome Atlas (TCGA) public database collecting a broad range of bladder cancer gene expression data, TCGA data mining has become an important tool for molecular analysis, such as tumour molecular subtyping and diagnostic or prognostic biomarker screening. By analysing gene expression profiles, a large number of new molecular biomarkers at the transcriptome level have been identified. Accordingly, studies on prognosis biomarkers of bladder cancer in recent years are primarily based on RNA expression. Long non-coding RNAs (a group of non-protein-coding transcripts longer than 200 nucleotides) and microRNAs (a class of small non-coding RNAs that have potential in RNA silencing) have been demonstrated to play essential roles in regulating gene expression at the transcriptional or post-transcriptional level and have prognostic value in multiple tumours (4-8). Concerning bladder cancer, accumulative studies have revealed the potential of lncRNAs or miRNAs as prognostic markers. The lncRNA HOTAIR has been demonstrated by Shang et al as an independent prognostic biomarker of overall survival in bladder transitional cell carcinoma patients (9). It was identified by $\mathrm{Wu}$ et al (10) that high tissue metastasis associated with lung adenocarcinoma transcript 1 (MALAT1) level was associated with an inferior clinical outcome in various cancers, including bladder cancer. Furthermore, Ratert et al (11) screened out and validated 15 bladder cancer-specific miRNAs, among which miR-141 and miR-205 were related to the overall survival time. miR-31, miR-100 and miR-145 were reported to be independent prognostic factors in patients with bladder cancer (12-14). Since the prognostic factors of bladder cancer are complex and collectively profiled lncRNAs or microRNAs have demonstrated considerable prognostic power, current studies are focused on prognostic signatures or combinations of multi-lncRNAs or multi-microRNAs. Dong et al performed a comprehensive analysis of 234 BLCA patients and demonstrated that a four-lncRNA (AC005682.5, CTD-2231H16.1, CTB-92J24.2 and RP11-727F15.13) signature could be a novel independent biomarker for predicting the survival of patients with BLCA (15). Zhou et al (16) identified an eight-miRNA signature, including three upregulated (miR-141, miR-200c and miR-21) and five downregulated (miR-145, miR-125, miR-199a, let-7c and miR-99a) miRNAs that could predict overall survival (OS) of bladder cancer. A four-miRNA (miR-422a-3p, miR-486-3p, miR-103a-3p and miR-27a-3p) signature was developed, and its considerable predictive potential was verified for muscle-invasive bladder cancer (17).

Compared with lncRNAs or microRNAs, protein-coding gene transcripts or mRNA levels directly reflect the gene expression level, and some studies have confirmed that PCGs can predict bladder cancer patient survival. For example, Mitra et al (18) identified a gene signature comprised of JUN, MAP2K6, STAT3 and ICAM1 that could predict the clinical outcome of bladder cancer patients. Another study found that a 24-gene hypoxia signature demonstrated strong and independent prognostic and predictive value for muscle invasive bladder cancer patients (19).

In conclusion, IncRNAs, microRNAs and PCGs can be prognostic markers of bladder cancer. However, there are no studies that explore the role of a multidimensional transcriptome signature combining lncRNAs and microRNAs with PCGs in bladder cancer. In the present study, we investigated the clinical value of a PCG-lncRNA-microRNA signature by mining the expression profiles of 239 bladder cancer patients.

\section{Materials and methods}

lncRNA, microRNA and mRNA expression data of BLCA patients. We downloaded microRNA (Illumina HiSeq microRNA Seq) and mRNA (Illumina HiSeq RNA Seq V2) level 3 expression data and corresponding clinical information of BLCA patients from the TCGA portal (https://genome-cancer. ucsc.edu/proj/site/hgHeatmap/). IncRNA expression data were obtained from the TANRIC database (http://ibl.mdanderson. org/tanric/_design/basic/index.html). Genes with missing expression values in $>30 \%$ of samples or patients were removed, and the remaining missing values were entered by the k-nearest neighbour method. Genes whose RPKM expression values were 0 in all samples were excluded (20). Following these steps, we obtained a total of 239 bladder cancer patients or samples for the present study. Since the data were obtained from TCGA, further approval by an Ethics Committee was not required. The present study met the publication guidelines provided by TCGA (http://cancergenome.nih.gov/publications/ publicationguidelines).

Construction of a prognostic PCG-lncRNA-microRNA signature in the training dataset. We used univariate Cox proportional hazards regression analysis to assess the relationship between the gene expression of lncRNAs, microRNAs and PCGs and patient OS in the training dataset. Considering that a smaller number of lncRNAs, microRNAs and PCGs in the model render the model more practical, we used the random survival forests-variable hunting (RSFVH) algorithm to filter out genes until three IncRNAs, three microRNAs and three PCGs were screened out (21).

Subsequently, in order to further screen prognostic genes with better predictive power, we performed a multivariable Cox regression analysis and developed a model to estimate prognosis risk as follows:

$$
\text { Risk Score }(\mathrm{RS})=\sum_{\mathrm{i}=1}^{\mathrm{N}}\left(\operatorname{Exp}_{\mathrm{i}} * \operatorname{Co\mathrm {e}}_{\mathrm{i}}\right)
$$

where $\mathrm{N}$ is the number of prognostic lncRNAs, microRNAs or PCGs; $\operatorname{Exp}_{\mathrm{i}}$ is the expression value of lncRNAs, microRNAs or PCGs; and $\mathrm{Coe}_{\mathrm{i}}$ is the estimated regression coefficient of lncRNAs, microRNAs or PCGs in the multivariable Cox regression analysis. Each patient then obtained 511 risk scores since three lncRNAs, three microRNAs and three PCGs could form $2^{9}-1=511$ combinations or signatures. The time-dependent receiver operating characteristic (ROC) curve was used to compare the sensitivity and specificity of the survival prediction risk score of the 511 signatures in the training dataset. AUC were calculated from the ROC curve. By comparing the AUC values, we constructed the prognostic PCG-lncRNA-microRNA signature in the training group.

Statistical analysis. We used the median risk score in the training dataset as a cut-off value (22), and the BLCA patients 
were divided into high- and low-risk groups. The estimation of survival time and comparison of survival curves of the high- and low-risk groups were obtained with the Kaplan-Meier survival analyses, and statistical significance was assessed using the two-sided log-rank test. We then validated the prognostic performance of the PCG-IncRNA-microRNA signature in the test dataset by the Kaplan-Meier survival analysis and ROC values. We compared the survival prediction power of the PCG-IncRNA-microRNA signature with TNM stage by the ROC values. Furthermore, multivariable Cox regression analysis and data stratification analysis were performed to assess whether the PCG-IncRNA-microRNA signature was an independent prognostic factor within the available data. $\mathrm{P}<0.05$ was considered to indicate a statistically significant difference. All analyses were performed using the R programme (www.r-project.org), including packages named pROC, survival and randomForestSRC downloaded from Bio-conductor.

Function prediction of the selected prognostic lncRNAs, microRNAs and PCGs. The co-expressed relationships between the selected prognostic genes ( 2 lncRNAs, 2 microRNAs and 2 PCGs) and protein-coding genes in BLCA patients were computed using Pearson correlation coefficient visualized by Cytoscape. Gene Ontology (GO) and Kyoto Encyclopedia of Genes and Genomes (KEGG) enrichment analyses of the co-expressed protein-coding genes were then performed to predict their biological function. GO analysis was performed in the ClueGo of the Cytoscape plugin (version 3.2.3) $(23,24)$, which is a commonly used functional annotation tool that can assess over-representation of functional categories among a gene set of interest. Enrichment analysis was performed using the functional annotation chart and functional annotation clustering options and was limited to GO terms and KEGG pathways in the 'Biological Process' categories. Functional annotation with $\mathrm{P}<0.05$ was considered significant.

\section{Results}

Patient characteristics and expression profiles. Expression profiles and corresponding clinical data of 239 (out of 411) patients diagnosed with BLCA were downloaded from TCGA database, and 172 (out of 411) patients with missing clinical data were not included in the present study. The median age of the enrolled patients was 69 years (60-76 years). Of the BLCA patients, 235 were stage I, II, III and IV disease, and the stage of 4 patients was unknown. Other clinical information of patient characteristics is summarized in Table I. Simultaneously, the expression values of 6,603 lncRNAs, 429 microRNAs and 14,644 PCGs for the BLCA patients were obtained after eliminating low-expression genes, and missing expression values were removed as described in the Materials and methods. All of these gene expression values were $\log 2$ transformed. Subsequently, we divided the dataset randomly into two groups (training dataset, $\mathrm{n}=119$; test dataset, $\mathrm{n}=120$ ) to explore and validate the prognostic PCG-IncRNA-microRNA signature of the BLCA patients. The selection process of the prognostic PCG-lncRNA-microRNA signatures is displayed in Fig. 1.

Identification of three prognostic IncRNAs, microRNAs and $m R N A s$ from the training dataset. We used the training dataset
Table I. Patient demographics and clinical characteristics.

\begin{tabular}{lccr}
\hline Characteristics & Training set & Testing set & Total \\
\hline pTNM stage & & & \\
Unknown & 3 & 1 & 4 \\
stage I & 1 & 1 & 2 \\
stage II & 28 & 48 & 76 \\
stage III & 34 & 46 & 80 \\
stage IV & 53 & 24 & 77 \\
Vital status & & & \\
Living & 76 & 82 & 158 \\
Dead & 43 & 38 & 81 \\
Sex & & & \\
Female & 32 & 25 & 57 \\
Male & 87 & 95 & 182 \\
Age (years) & & & \\
$>69$ & 47 & 52 & 99 \\
$\leq 69$ & 72 & 68 & 140 \\
\hline
\end{tabular}

to select the prognostic genes. Firstly, using survival time and clinical outcome as the dependent variable, we performed univariate Cox proportional hazards regression analysis of all of the lncRNAs, microRNAs and PCGs expressed in the training dataset and identified a 2,974-gene set including 568 lncRNAs, 77 microRNAs and 2,339 PCGs, which were significantly correlated with patient OS (P-value $<0.05$, data not shown). To visually display these selected genes, we drew a volcano plot using the univariate Cox coefficient as the $\mathrm{x}$-axis and $-\log _{10}$ (P-value) as the y-axis. As displayed in Fig. 2A, blue dots in the volcano plot represent the 2,974 genes with significant differences $(\mathrm{P}<0.05)$, and red dots represent the remaining genes with no significant differences. Secondly, we further screened the prognostic genes from the above 2,974 genes using a random forest supervised classification algorithm, and three lncRNAs (RP11-60L3.1, CTD-3195I5.3 and TMC4), three microRNAs (hsa-miR-3913-1, hsa-miR-891a and hsa-mir-1976) and three PCGs (ACADS, C1QTNF9B and HP) strongly related to patient survival were screened out according to the permutation important score by the random survival forests-variable hunting (RSFVH) algorithm.

Construction of the prognostic PCG-IncRNA-microRNA signature in the training dataset. Three $\operatorname{lncRNAs}$, three microRNAs and three PCGs formed 511 combinations or signatures, and each combination had a risk score according to the risk score model constructed in the Materials and methods. To select a signature with the biggest prediction power from these 511 combinations in the training dataset, we performed 511 time-dependent ROC analyses using the patient survival statuses and signature risk scores as variables and compared their areas under the respective ROC curves (AUC) (data not shown). Among them, the PCG-IncRNA-microRNA combination comprised of ACADS, C1QTNF9B, RP11-60L3.1, CTD-3195I5.3, has-miR-3913-1 and has-miR-891a with the max AUC was screened out 


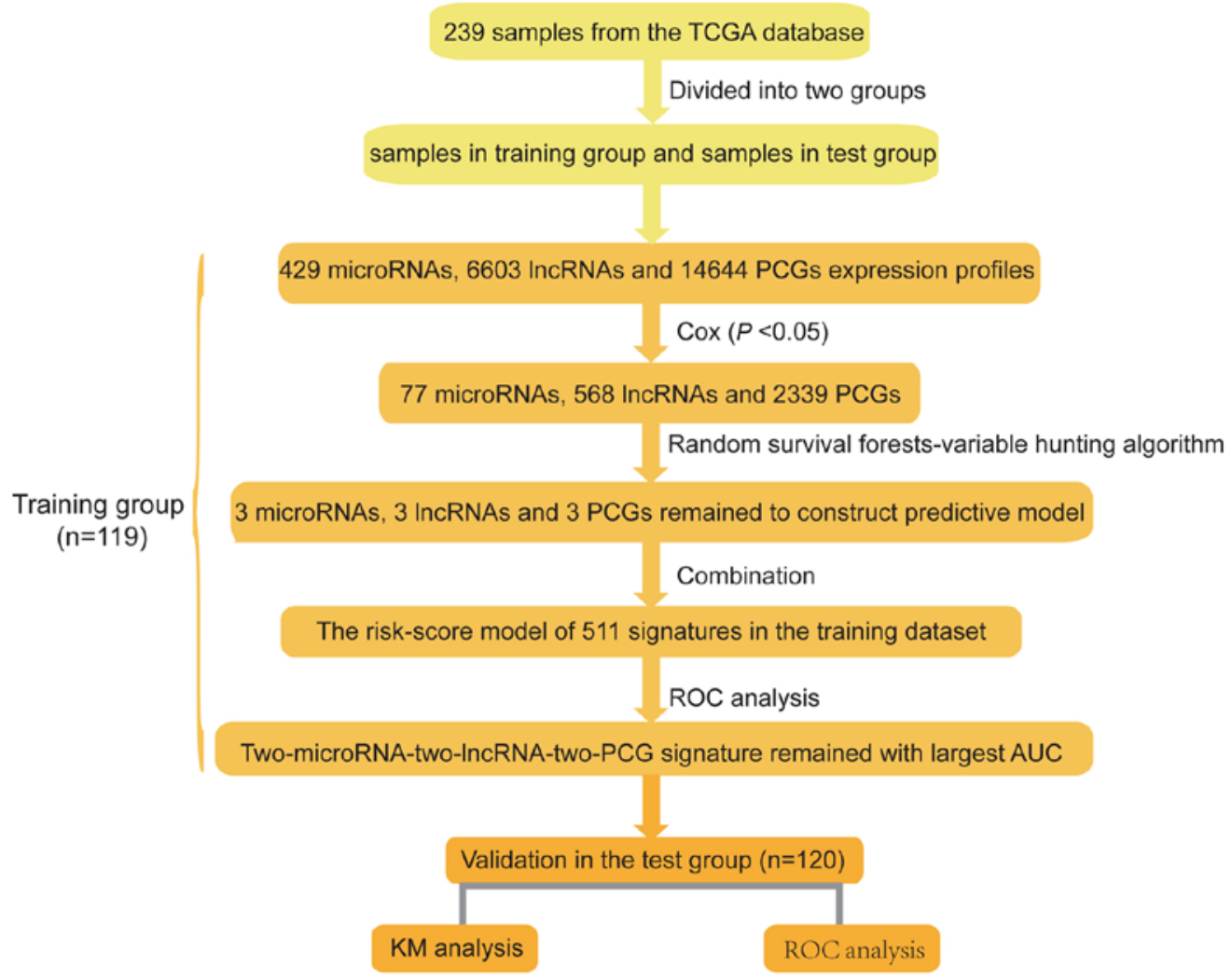

Figure 1. Schedule of the present study. The order of the analyses to construct the signature risk score model and validate its prognostic power.
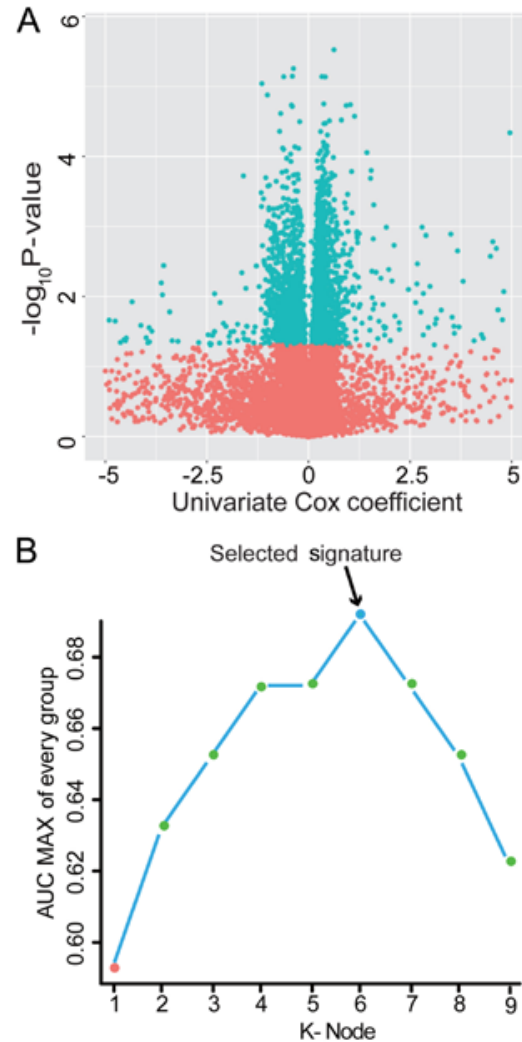

Figure 2. Identification of the prognostic PCG-IncRNA-microRNA signature in the training dataset. (A) Univariate Cox proportional hazards regression analysis of the IncRNAs, microRNAs and PCGs expression profile data in the training dataset. (B) The prognostic signature with the biggest prediction power was screened out. Important scores to filter genes and the accuracies of all 511 signatures were calculated, and the nine highest accuracies for $\mathrm{k}=1,2 \ldots . .9$ are shown in the plot.
(Fig. 2B and Table II). The risk score model comprised of ACADS, C1QTNF9B, RP11-60L3.1, CTD-3195I5.3, has-miR-3913-1 and has-miR-891a was as follows: Risk score $=(-0.59 \times$ expression value of ACADS $)+(0.47 \mathrm{x}$ expression value of C1QTNF9B $)+(0.11 \mathrm{x}$ expression value of RP11-60L3.1) + (-1.54 x expression value of CTD-3195I5.3) $+(-0.46 \mathrm{x}$ expression value of hsa-miR-3913-1) $+(0.38 \mathrm{x}$ expression value of hsa-miR-891a). The AUC of the selected PCG-IncRNA-microRNA signature in the training group was 0.79 , demonstrating its good survival prediction performance.

Validation of the selected PCG-IncRNA-microRNA signature for survival prediction in the training and the test dataset. In the training dataset, the selected PCG-IncRNA-microRNA signature gave each patient a risk score with the prognostic model and divided patients into a high-risk group $(\mathrm{n}=60)$ and low-risk group $(n=59)$. The median risk score was used as the cut-off point. Then, Kaplan-Meier survival analysis was performed to compare the overall survival rates of the two groups of patients. Patients in the high-risk group had significantly shorter OS than those in the low-risk group (median survival: 16.9 months vs. 54.9 months, log-rank test $\mathrm{P}<0.001$; Fig. $3 \mathrm{~A}$ ). The 5 -year OS rate of the patients in the high-risk group was $<11 \%$, while that of the patients in the low-risk group was $>60 \%$.

To validate the prognostic prediction power of the signature, the same prognostic risk score model obtained from the training dataset was used to calculate the PCG-IncRNA-microRNA signature-based risk scores of 120 patients in the test dataset. Similarly, the test dataset was divided into two groups using the same median cut-off point obtained from the training dataset: One group with a high risk and the other group with a low risk. 
Table III. Association of the PCG-IncRNA-microRNA signature with clinicopathological characteristics in BLCA patients (training group, $\mathrm{n}=119$ ).

\begin{tabular}{|c|c|c|c|}
\hline \multirow[b]{2}{*}{ Variables } & \multicolumn{2}{|c|}{ PCG-IncRNA signature } & \multirow[b]{2}{*}{ P-value } \\
\hline & Low risk ${ }^{\mathrm{a}}$ & High risk $^{\mathrm{a}}$ & \\
\hline Age & & & 0.76 \\
\hline$>69$ & 28 & 19 & \\
\hline$\leq 69$ & 32 & 40 & \\
\hline Sex & & & 1.00 \\
\hline Female & 16 & 16 & \\
\hline Male & 44 & 43 & \\
\hline Pathologic M & & & 0.84 \\
\hline M0 & 32 & 31 & \\
\hline M1 & 1 & 2 & \\
\hline MX & 27 & 26 & \\
\hline Regional lymph nodes & & & 0.01 \\
\hline No & 32 & 21 & \\
\hline N1 & 5 & 12 & \\
\hline $\mathrm{N} 2$ & 12 & 21 & \\
\hline N3 & 1 & 3 & \\
\hline NX & 10 & 2 & \\
\hline Primary tumor & & & 0.11 \\
\hline $\mathrm{T} 1$ & 1 & 0 & \\
\hline $\mathrm{T} 2$ & 15 & 14 & \\
\hline T3 & 26 & 37 & \\
\hline $\mathrm{T} 4$ & 10 & 6 & \\
\hline $\mathrm{TX}$ & 8 & 2 & \\
\hline pTNM stage & & & 0.02 \\
\hline I & 1 & 0 & \\
\hline II & 19 & 9 & \\
\hline III & 20 & 14 & \\
\hline IV & 18 & 35 & \\
\hline stage $X$ & 2 & 1 & \\
\hline
\end{tabular}

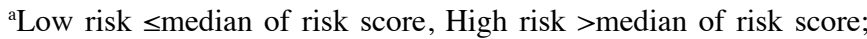
Chi-square test, $\mathrm{P}<0.05$ was considered significant. Stage $\mathrm{X}$, unknown stage.

Kaplan-Meier curves for the high- and low-risk groups in the test dataset are displayed in Fig. 3B. Similar to the results of the training dataset, the OS rate of the high-risk group in the test dataset was significantly lower than that of the low-risk group (median survival: 18.2 months vs. 58.9 months, log-rank test $\mathrm{P}=0.003)$. The $\mathrm{OS}$ rate of the patients in the high-risk group was $\sim 19 \%$ at 5 years in contrast to $45 \%$ in the low-risk group.

The selected PCG-lncRNA-microRNA signature is an independent prognostic factor. To obtain a better understanding of the clinical significance of the PCGlncRNA-microRNA signature in BLCA patients, we correlated the signature to a series of clinicopathological parameters in the training groups $(n=119)$. As displayed in Table III, there 

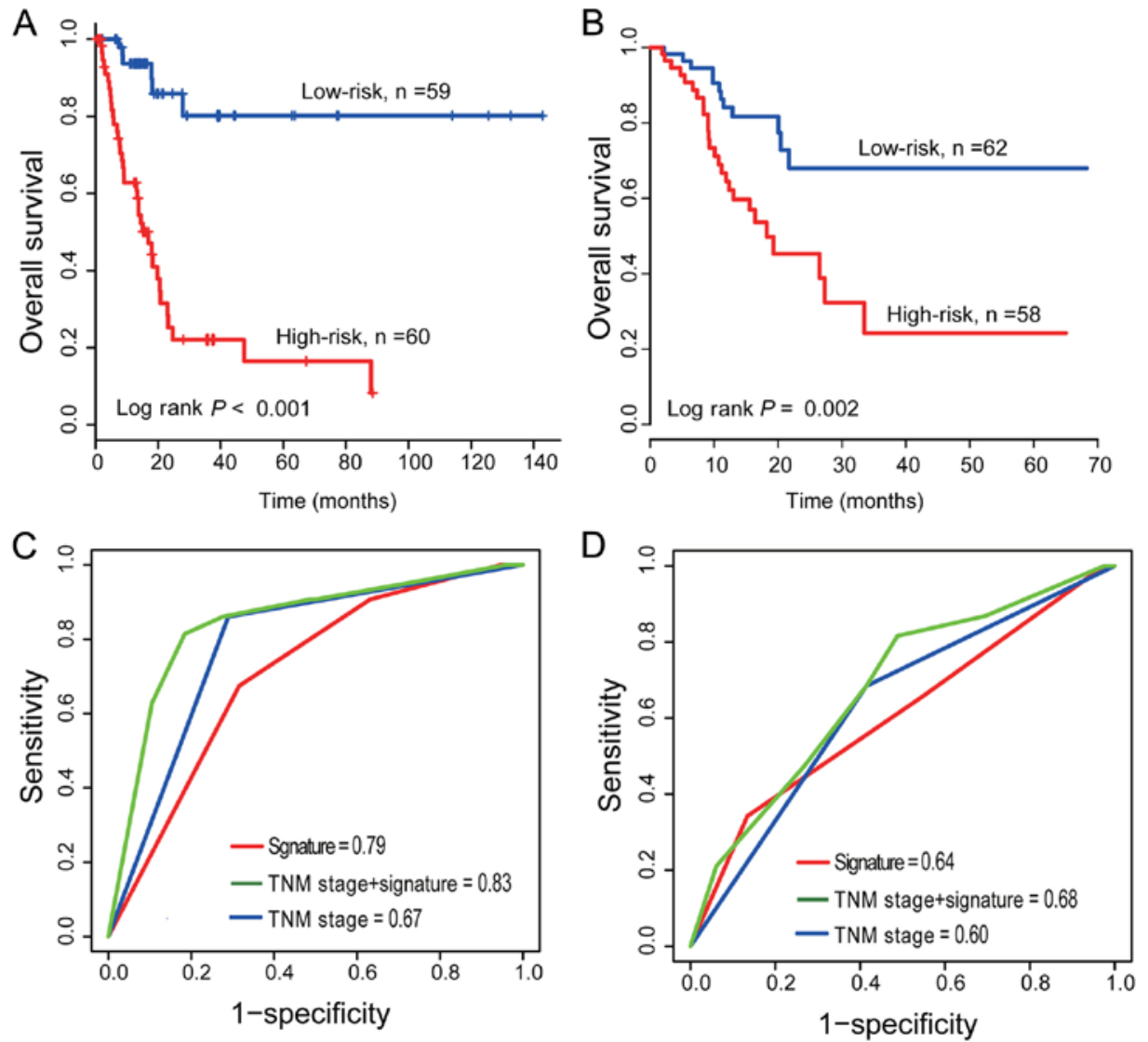

Figure 3. PCG-IncRNA-microRNA signature predicts overall survival of patients with BLCA and comparison to the survival prediction power of the PCG-lncRNA-microRNA signature and TNM stage. (A and B) Kaplan-Meier survival curves classify patients into high- and low-risk groups by the PCG-IncRNA-microRNA signature in the training and test dataset. P-values were calculated by log-rank test. (C and D) ROC analysis was used to compare the survival prediction power between the PCG-IncRNA-microRNA signature and the TNM stage in the training and test datasets.

was an association between the PCG-lncRNA-microRNA signature and clinicopathological variables, including regional lymph nodes and TNM stage (Chi-square test, $\mathrm{P}<0.05$ ).

To assess whether the PCG-IncRNA-microRNA signature was an independent risk factor for survival prediction, multivariable Cox regression analysis was performed using the PCG-lncRNA-microRNA signature-based risk score and other clinical features as covariates. The P-values of the prognostic signature in multivariable Cox regression analysis from the training datasets was $<0.05$, which indicated that the PCG-lncRNA-microRNA signature risk score was an independent prognostic factor, following adjustment for other clinical features including sex, age and pTNM (high-risk group vs. low-risk group, HR=7.42, 95\% CI 3.08-17.88, $\mathrm{P}<0.001, \mathrm{n}=119$; Table IV). The same result appeared in the test dataset $(\mathrm{HR}=2.84,95 \%$ CI 1.43-5.64, $\mathrm{P}<0.001, \mathrm{n}=120$; Table IV).

Comparison of the survival prediction power of the PCG-lncRNA-microRNA signature with TNM stage. To compare the survival prediction power of TNM stage and the PCG-lncRNA-microRNA signature, we performed ROC analysis considering that a larger AUC usually represented a better model for prediction $(25,26)$. In the training dataset $(n=119)$, the AUC of the PCG-IncRNA-microRNA signature was greater than that of the TNM stage $\left(\mathrm{AUC}_{\mathrm{Signature}}=0.79 \mathrm{vs}\right.$. $\mathrm{AUC}_{\mathrm{TNM}}=0.67$, $\mathrm{n}=119$; Fig. $3 \mathrm{C}$ ), which demonstrated that the signature for

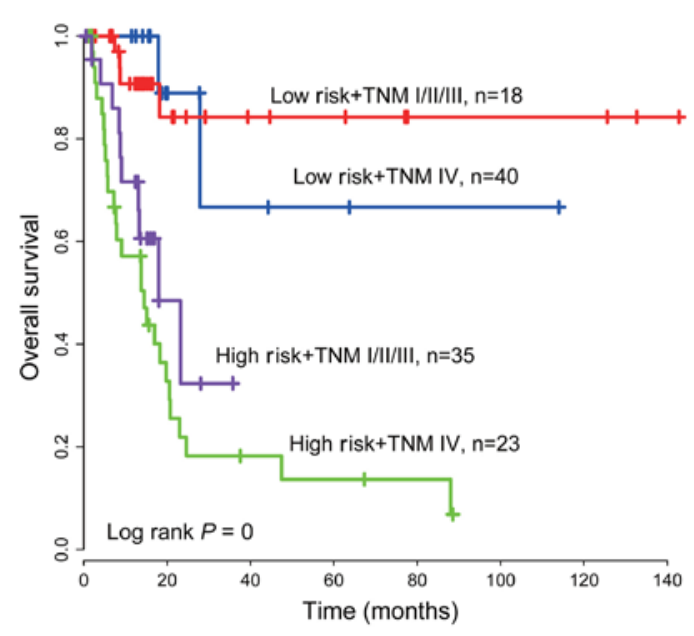

Figure 4. Kaplan-Meier analysis for the signature combined with TNM stage in the training group.

survival prediction in our study had high sensitivity and specificity and had important clinical significance. The same result was indicated in the test group $\left(\mathrm{AUC}_{\text {Signature }}=0.64 \mathrm{vs}\right.$. $\mathrm{AUC}_{\mathrm{TNM}}=0.60, \mathrm{n}=120$; Fig. 3D), while the model combining TNM with the PCG-IncRNA-microRNA signature had a larger AUC than the TNM stage or signature alone $\left(\mathrm{AUC}_{\mathrm{TNM}+}\right.$ Signature $=0.83 / 0.68$ in the training/test group; Fig. $3 \mathrm{C}$ and D). 
Stratification analysis. Due to the correlation between the TNM stage and the PCG-lncRNA-microRNA signature $(\mathrm{P}<0.05$, Table III) and the combination of TNM with the PCG-lncRNA-microRNA signature having a relatively large AUC, we stratified the TNM stage by the PCG-lncRNA-microRNA signature risk score, and thus, patients from the training dataset $(n=119)$ were grouped into four groups: high-risk and TNM IV stage, high-risk and TNM I+II+III stage, low-risk and TNM IV stage, and low-risk and TNM I+II+III stage. The Kaplan-Meier test was performed, and Kaplan-Meier curves revealed that the combination model could more precisely subdivide patients. The log-rank test revealed that the signature could further subdivide TNM I+II+III stage patients into either a high-risk group with shorter survival or a low-risk group with longer survival (log-rank test $\mathrm{P}<0.001)$. Similarly, the TNM stage IV patients were also divided into a high-risk group with lower OS and a low-risk group with higher OS (log-rank test $\mathrm{P}<0.001$, Fig. 4).

Functional characterization of the selected prognostic lncRNAs, microRNAs and PCGs. Co-expression relationships of these selected IncRNAs, microRNAs and PCGs with their corresponding protein-coding genes were computed using Pearson's correlation coefficient in the training/test dataset. A total of 1,593/1,643 protein-coding genes in the training/test dataset were highly correlated with at least one of the selected lncRNAs, microRNAs and PCGs, and the interaction network was visualized by Cytoscape (27) (Pearson's correlation coefficient $>0.40, \mathrm{P}<0.05$; Fig. 5A and data not shown). GO and KEGG of these co-expressed protein-coding genes was performed, indicating that in the two datasets co-expressed protein-coding genes were significantly enriched in 31 different GO terms and KEGG pathways $(\mathrm{P}<0.05)$. The six genes may be involved in tumourigenesis by interacting with those protein-coding genes that affect the important biological processes such as cellular nitrogen compound metabolic process, nucleobase-containing compound metabolic process, heterocycle metabolic process and cellular aromatic compound metabolic process (top 5 ranking by P-value, Fig. 5B).

\section{Discussion}

In a broad sense, bladder cancer includes not only bladder urothelial carcinoma (BLCA) but also non-epithelial cancers, such as lymphoma or sarcoma. However, BLCA is ordinarily considered as bladder cancer since it accounts for $90 \%$ of bladder cancers. In the present study, we used BLCA samples to explore bladder cancer prognosis markers. In terms of prognosis, bladder cancer patients with similar TNM staging have different survival time. A portion of them may encounter disease recurrence and progression, which decreases the OS rate to a great extent. Thus, the 5-year survival rate of BLCA patients varies significantly from 5 to $70 \%$. Thereby, TNM staging, as the dominant prognostic assessment tool, is not adequate for prediction of individual outcomes. In the era of precision medicine, gene signature has been identified as being able to serve as a novel biomarker for predicting the survival of various carcinomas. In the past decades, with the development of transcriptomic and bioinformatics studies, mRNAs as well

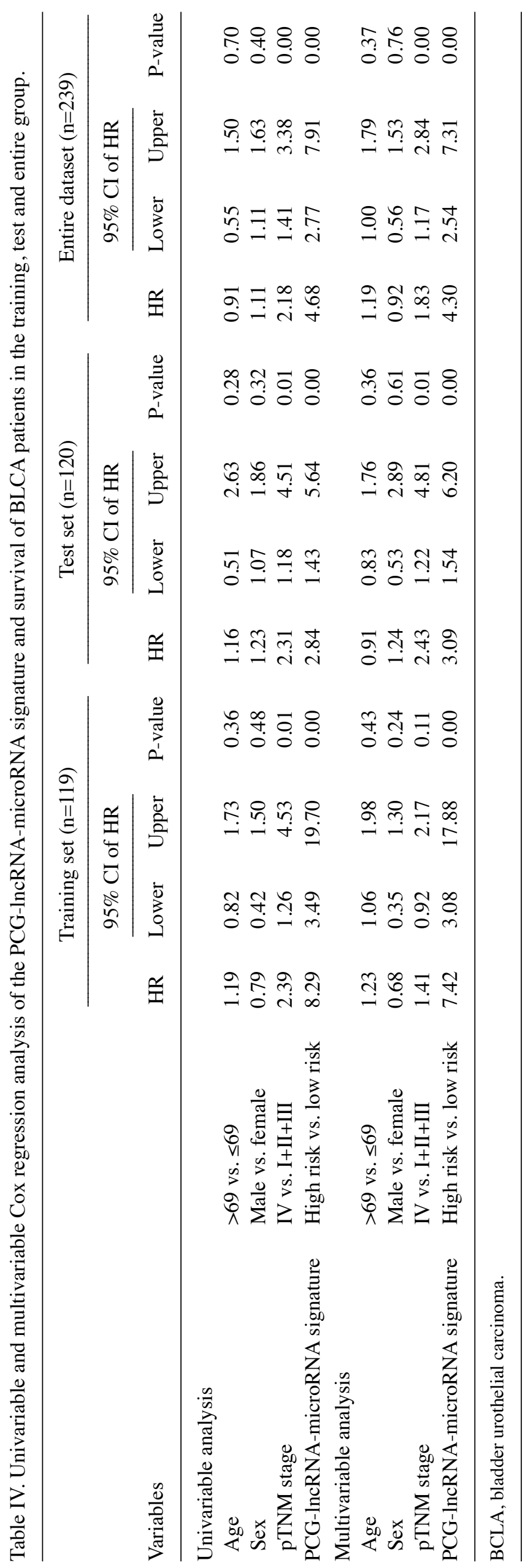




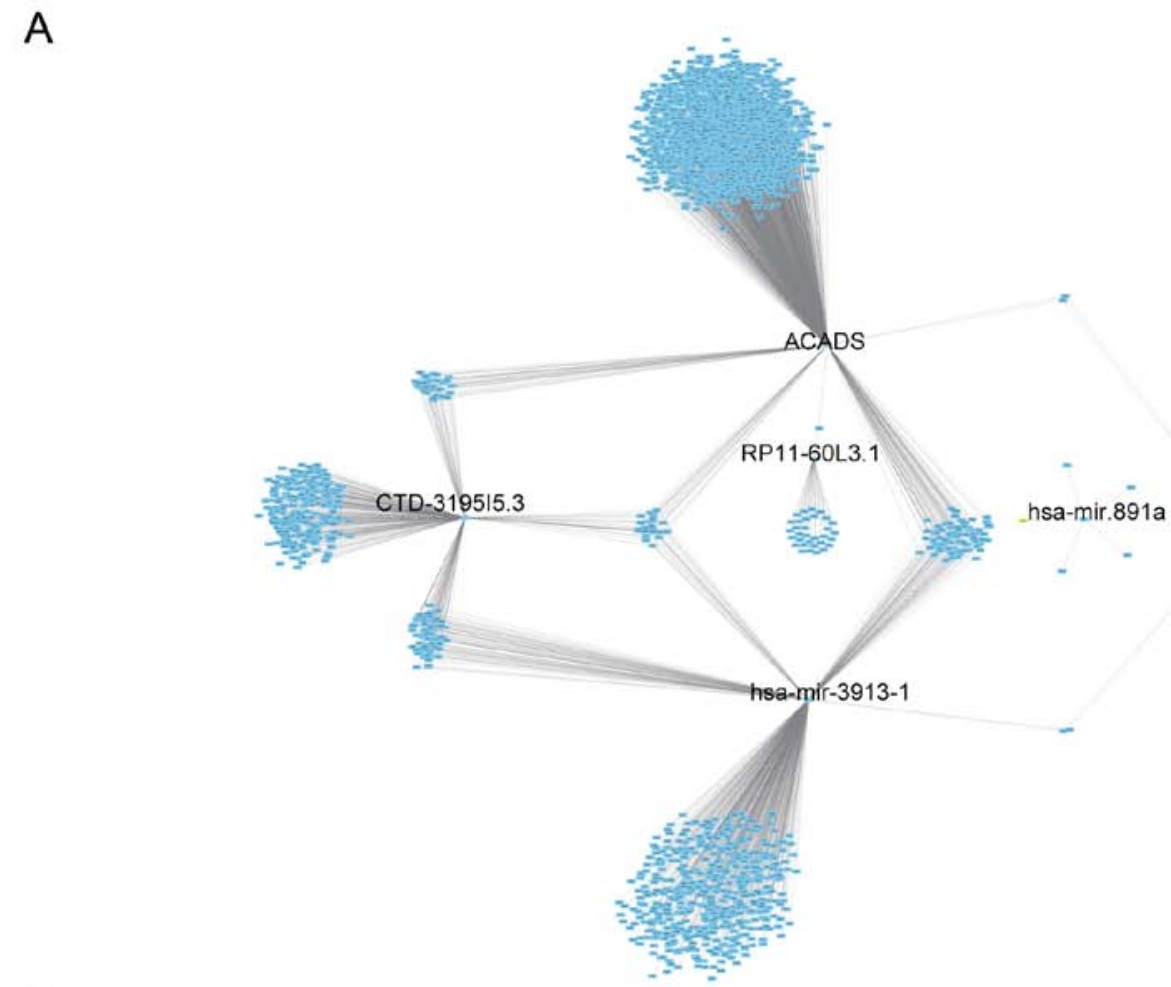

B
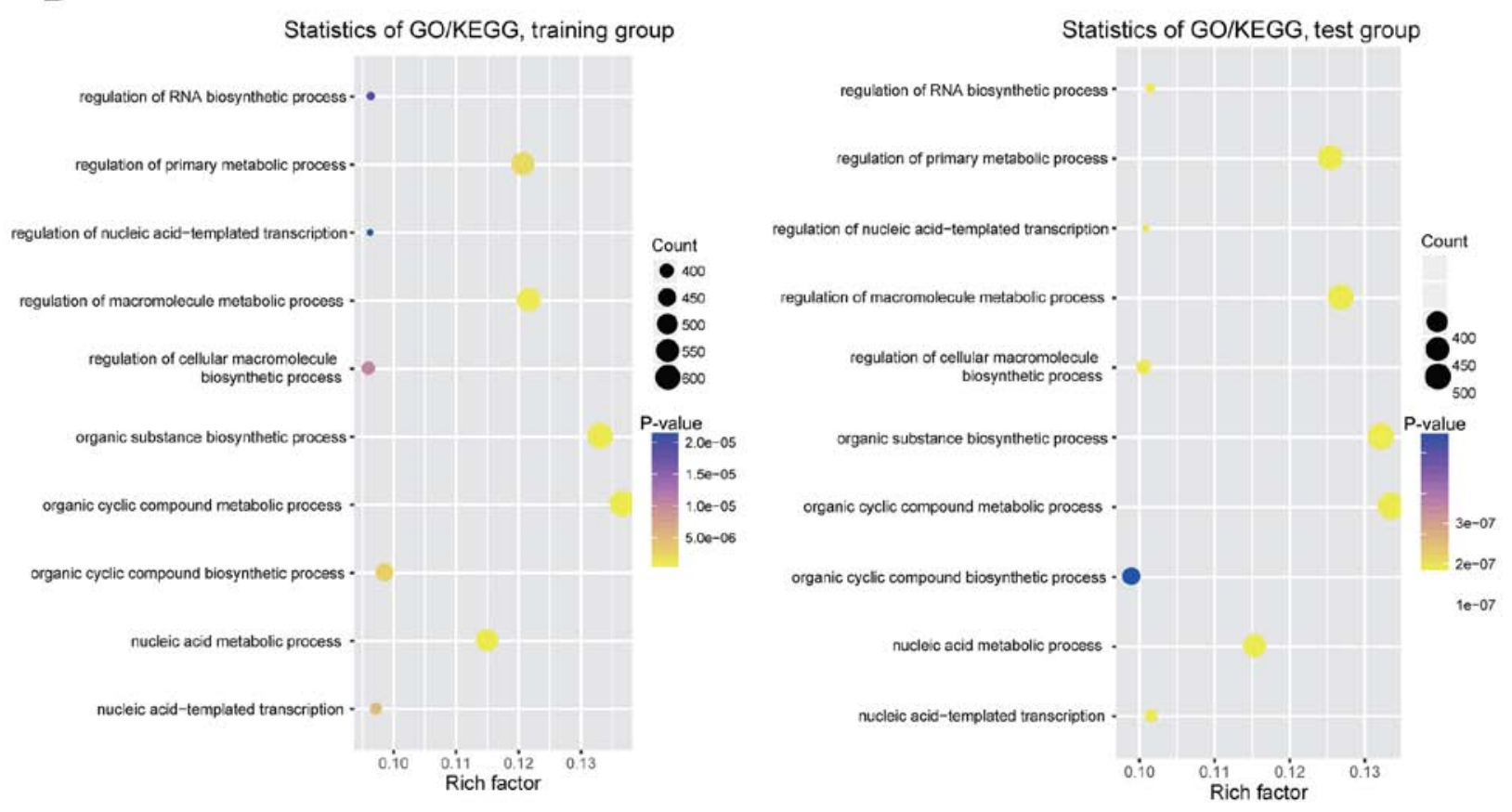

Figure 5. (A) Co-expression network and (B) functional enrichment of the co-expressed protein-coding genes with two prognostic lncRNAs, microRNAs and PCGs in the training and test groups.

as non-coding RNAs including lncRNAs and microRNAs, have become the focus of tumour prognosis-related research.

lncRNAs have been found to be involved in regulating gene expression at transcriptional, post-transcriptional and epigenetic levels (4), and microRNAs could regulate hundreds of downstream genes by targeting the 3'untranslated region of specific messenger RNAs for degradation or translational repression (4). Furthermore, similar to protein coding genes or mRNAs, accumulating evidence has indicated that both
lncRNAs and microRNAs are involved in oncogenic and tumour suppressive pathways and perform various functions in a wide variety of important biological processes, such as transcriptional regulation, cell growth and tumourigenesis (29-33). Different from protein coding genes, lncRNAs and microRNAs are tissue-specific and their expression is more closely associated with biological function and tumour status as their non-coding feature (30,34-37). All these characteristics open a door for lncRNAs, microRNAs and PCGs 
to be used as cancer diagnostic or prognostic biomarkers. Their prognostic value in various types of cancer has been confirmed by numerous studies. As aforementioned, lncRNAs, microRNAs and PCGs have revealed their prognostic potential in bladder cancer. However, current studies are limited to one type of RNA (lncRNA, microRNA or mRNA) or RNA signature, and there is little research combining three types of RNA and exploring more powerful markers from a comprehensive and multidimensional transcriptome level. In the present study, by analysing the BLCA transcriptome data, we aimed to find a multidimensional transcriptome gene signature that could predict the survival of bladder cancer patients. Based on the results of TCGA expression data mining, we identified a signature involving two IncRNAs, two microRNAs and two PCGs that was significantly associated with the OS of BLCA patients in the training dataset. The selected signature separated BLCA patients into a low-risk group and a high-risk group with significantly different survival times in the training or test dataset, demonstrating its good prognostic performance. Multivariable Cox regression analysis was performed to assess the independence of the selected PCG-IncRNA-microRNA signature in predicting OS in the training dataset and test dataset. With sex and pTNM stage as covariables in the regression analysis, risk score of the PCG-IncRNA-microRNA signature maintained an independent correlation with OS. Collectively, these results indicated that the prognostic power of the PCG-IncRNA-microRNA signature was independent of other clinical features. In addition, comparison of the prediction ability of TNM staging and the multidimensional transcriptome signature confirmed that the signature was superior to TNM staging. The stratification analysis found that the PCG-IncRNA-microRNA signature combined with TNM stage demonstrated a more robust prognostic power, implying that the signature could be an auxiliary biomarker of TNM stage to more precisely subdivide BLCA patients.

As for the characteristics of the PCGs, IncRNAs and microRNAs in the signature, we found that high expression of C1QTNF9B, RP11-60L3.1 and hsa-miR-891a was associated with a short survival time (univariable Cox coefficient $>0$ ), and high expression of ACADS, CTD-3195I5.3 and hsa-miR-3913-1 was associated with a long survival time (univariable Cox coefficient $<0$ ) (the KM analysis of the six genes in the training group are data not shown). C1QTNF9B is a novel protein that hetero-oligomerizes with $\mathrm{C} 1 \mathrm{q} / \mathrm{TNF}$ family members. C1q/TNF family members have been revealed to play diverse roles in various physiological processes in different tissue compartments, ranging from development to the immune, endocrine, skeletal, neuronal, reproductive, sensory and vascular systems (38). ACADS is involved in free fatty acid $\beta$-oxidation and regulates energy homeostasis (39). hsa-miR-891a can target bladder cancer-associated protein (BLCAP) potentially in the 3'UTR (40), while there is a lack of research for the remaining genes. Although functions of these IncRNAs, microRNAs and PCGs have been inferred by bioinformatics analysis, the biological roles of the selected genes in tumourigenesis are still not clear and should be investigated in further experimental studies. With the rapidly increasing related studies, more multidimensional signatures will become available, such as combining PCGs, IncRNAs and microRNAs with circular RNAs or other non-coding RNAs.
Limitations in the present study need to be acknowledged. First, only a fraction of human mRNAs (14,644 out of 30,000+), lncRNAs $(6,603$ out of $15,000+)$ and microRNAs (429 out of $2,000+)$ were included in the present study. Therefore, we may have missed candidates that are potentially correlated with BLCA overall survival. Second, we failed to further seek the mechanisms behind the prognostic values of these PCGs, IncRNAs and microRNAs in BLCA, and experimental studies on these PCGs, IncRNAs and microRNAs may provide important information to deepen our understanding of their functional roles. Finally, although we demonstrated the selected PCG-IncRNA-microRNA signature may replace or complement the TNM staging, applying it for clinical use still warrants more confirmed studies. Despite these drawbacks, however, the PCG-IncRNA-microRNA signature has been demonstrated to serve as a marker for BLCA patient prognosis prediction.

In conclusion, the multidimensional transcriptome signature comprised of ACADS, C1QTNF9B, RP11-60L3.1, CTD-3195I5.3, hsa-miR-3913-1 and hsa-miR-891a can predict the survival of bladder cancer patients with more accuracy and further subdivide BLCA patients combined with TNM stages, revealing that the model has promising clinical significance.

\section{Acknowledgements}

Not applicable.

\section{Funding}

The present study was supported by the Bioinformatics Research Group of the Chinese Academy of Science.

\section{Availability of data and materials}

The datasets used during the present study are available from the corresponding author upon reasonable request.

\section{Authors' contributions}

CPL, JHZ, JL and SCZ collected, analysed, interpreted and drafted the data. JCG designed and supervised the study and provided the final approval of the manuscript. JHZ, JL and JCG provided technical support. All authors read and approved the manuscript and agree to be accountable for all aspects of the research in ensuring that the accuracy or integrity of any part of the work are appropriately investigated and resolved.

\section{Ethics approval and consent to participate}

Not applicable.

\section{Patient consent for publication}

Not applicable.

\section{Competing interests}

The authors declare that they have no competing interests. 


\section{References}

1. Clark PE, Spiess PE, Agarwal N, Bangs R, Boorjian SA, Buyyounouski MK, Efstathiou JA, Flaig TW, Friedlander T, Greenberg RE, et al: NCCN Guidelines Insights: Bladder cancer, version 2.2016. J Natl Compr Canc Netw 14: 1213-1224, 2016.

2. Siegel RL, Miller KD and Jemal A: Cancer Statistics, 2017. CA Cancer J Clin 67: 7-30, 2017.

3. Solomon JP and Hansel DE: Prognostic factors in urothelial carcinoma of the bladder: Histologic and molecular correlates. Adv Anat Pathol 22: 102-112, 2015.

4. Dykes IM and Emanueli C: Transcriptional and post-transcriptional gene regulation by long non-coding RNA. Genomics Proteomics Bioinformatics 15: 177-186, 2017.

5. Petrovic N, Ergun S and Isenovic ER: Levels of microRNA heterogeneity in cancer biology. Mol Diagn Ther 21: 511-523, 2017.

6. Adams BD, Parsons C, Walker L, Zhang WC and Slack FJ: Targeting noncoding RNAs in disease. J Clin Invest 127: 761-771, 2017.

7. Guo JC, Li CQ, Wang QY, Zhao JM, Ding JY, Li EM and $\mathrm{Xu}$ LY: Protein-coding genes combined with long non-coding RNAs predict prognosis in esophageal squamous cell carcinoma patients as a novel clinical multi-dimensional signature. Mol Biosyst 12: 3467-3477, 2016.

8. Guo JC, Wu Y, Chen Y, Pan F, Wu ZY, Zhang JS, Wu JY, $\mathrm{Xu} \mathrm{XE}$, Zhao JM, Li EM, et al: Protein-coding genes combined with long noncoding RNA as a novel transcriptome molecular staging model to predict the survival of patients with esophageal squamous cell carcinoma. Cancer Commun 38: 4, 2018.

9. Shang C, Guo Y, Zhang H and Xue YX: Long noncoding RNA HOTAIR is a prognostic biomarker and inhibits chemosensitivity to doxorubicin in bladder transitional cell carcinoma. Cancer Chemother Pharmacol 77: 507-513, 2016.

10. Wu Y, Lu W, Xu J, Shi Y, Zhang H and Xia D: Prognostic value of long non-coding RNA MALAT1 in cancer patients. Tumour Biol 37: 897-903, 2016.

11. Ratert N, Meyer HA, Jung M, Lioudmer P, Mollenkopf HJ, Wagner I, Miller K, Kilic E, Erbersdobler A, Weikert S, et al: miRNA profiling identifies candidate mirnas for bladder cancer diagnosis and clinical outcome. J Mol Diagn 15: 695-705, 2013.

12. Wang S, Li Q, Wang K, Dai Y, Yang J, Xue S, Han F, Zhang Q, Liu J and Wu W: Decreased expression of microRNA-31 associates with aggressive tumor progression and poor prognosis in patients with bladder cancer. Clin Transl Oncol 15: 849-854, 2013.

13. Wang S, Xue S, Dai Y, Yang J, Chen Z, Fang X, Zhou W, Wu W and Li Q: Reduced expression of microRNA-100 confers unfavorable prognosis in patients with bladder cancer. Diagn Pathol 7: 159,2012.

14. Villadsen SB,Bramsen JB, Ostenfeld MS, WiklundED,Fristrup N Gao S, Hansen TB, Jensen TI, Borre M, Ørntoft TF, et al: The miR-143/-145 cluster regulates plasminogen activator inhibitor-1 in bladder cancer. Br J Cancer 106: 366-374, 2012.

15. Bao Z, Zhang W and Dong D: A potential prognostic lncRNA signature for predicting survival in patients with bladder urothelial carcinoma. Oncotarget 8: 10485-10497, 2017.

16. Zhou H, Tang K, Xiao H, Zeng J, Guan W, Guo X, Xu H and Ye Z: A panel of eight-miRNA signature as a potential biomarker for predicting survival in bladder cancer. J Exp Clin Cancer Res 34: 53, 2015

17. Jiang X, Du L, Duan W, Wang R, Yan K, Wang L, Li J, Zheng G, Zhang X, Yang Y, et al: Serum microRNA expression signatures as novel noninvasive biomarkers for prediction and prognosis of muscle-invasive bladder cancer. Oncotarget 7: 36733-36742, 2016

18. Mitra AP, Pagliarulo V, Yang D, Waldman FM, Datar RH, Skinner DG, Groshen S and Cote RJ: Generation of a concise gene panel for outcome prediction in urinary bladder cancer. J Clin Oncol 27: 3929-3937, 2009.

19. Yang L, Taylor J, Eustace A, Irlam JJ, Denley H, Hoskin PJ, Alsner J, Buffa FM, Harris AL, Choudhury A, et al: A gene signature for selecting benefit from hypoxia modification of radiotherapy for high-risk bladder cancer patients. Clin Cancer Res 23: 4761-4768, 2017.
20. Xu J, Li Y, Lu J, Pan T, Ding N, Wang Z, Shao T, Zhang J, Wang $\mathrm{L}$ and $\mathrm{Li} \mathrm{X}$ : The mRNA related ceRNA-ceRNA landscape and significance across 20 major cancer types. Nucleic Acids Res 43: 8169-8182, 2015.

21. Li J, Chen Z, Tian L, Zhou C, He MY, Gao Y, Wang S, Zhou F, Shi S, Feng X, et al: LncRNA profile study reveals a three-lncRNA signature associated with the survival of patients with oesophageal squamous cell carcinoma. Gut 63: 1700-1710, 2014.

22. Zhou M, Guo M, He D, Wang X, Cui Y, Yang H, Hao D and Sun J: A potential signature of eight long non-coding RNAs predicts survival in patients with non-small cell lung cancer. J Transl Med 13: 231, 2015.

23. Bindea G, Mlecnik B, Hackl H, Charoentong P, Tosolini M, Kirilovsky A, Fridman WH, Pagès F, Trajanoski Z and Galon J: ClueGO: A Cytoscape plug-in to decipher functionally grouped gene ontology and pathway annotation networks. Bioinformatics 25: 1091-1093, 2009.

24. Guo JC, Xie YM, Ran LQ, Cao HH, Sun C, Wu JY, Wu ZY, Liao LD, Zhao WJ, Fang WK, et al: L1CAM drives oncogenicity in esophageal squamous cell carcinoma by stimulation of ezrin transcription. J Mol Med 95: 1355-1368, 2017.

25. Tse LA, Dai J, Chen M, Liu Y, Zhang H, Wong TW, Leung CC, Kromhout H, Meijer E, Liu S, et al: Prediction models and risk assessment for silicosis using a retrospective cohort study among workers exposed to silica in China. Sci Rep 5: 11059, 2015.

26. Heagerty PJ, Lumley T and Pepe MS: Time-dependent ROC curves for censored survival data and a diagnostic marker. Biometrics 56: 337-344, 2000.

27. Shannon P, Markiel A, Ozier O, Baliga NS, Wang JT, Ramage D, Amin N, Schwikowski B and Ideker T: Cytoscape: A software environment for integrated models of biomolecular interaction networks. Genome Res 13: 2498-2504, 2003.

28. He L and Hannon GJ: MicroRNAs: Small RNAs with a big role in gene regulation. Nat Rev Genet 5: 522-531, 2004.

29. Kornienko AE, Guenzl PM, Barlow DP and Pauler FM: Gene regulation by the act of long non-coding RNA transcription. BMC Biol 11: 59, 2013.

30. Fatica A and Bozzoni I: Long non-coding RNAs: New players in cell differentiation and development. Nat Rev Genet 15: 7-21, 2014.

31. Zhang XQ, Sun S, Lam KF, Kiang KM, Pu JK, Ho AS, Lui WM, Fung CF, Wong TS and Leung GK: A long non-coding RNA signature in glioblastoma multiforme predicts survival. Neurobiol Dis 58: 123-131, 2013.

32. Wan $\mathrm{J}, \mathrm{Wu} \mathrm{W}$, Che $\mathrm{Y}$, Kang $\mathrm{N}$ and Zhang R: Insights into the potential use of microRNAs as a novel class of biomarkers in esophageal cancer. Dis Esophagus 29: 412-420, 2016.

33. Geng Q, Fan T, Zhang B, Wang W, Xu Y and Hu H: Five microRNAs in plasma as novel biomarkers for screening of early-stage non-small cell lung cancer. Respir Res 15: 149, 2014.

34. Hauptman $\mathrm{N}$ and Glavac D: Long non-coding RNA in cancer. Int J Mol Sci 14: 4655-4669, 2013

35. Du Z, Fei T, Verhaak RG, Su Z, Zhang Y, Brown M, Chen Y and Liu XS: Integrative genomic analyses reveal clinically relevant long noncoding RNAs in human cancer. Nat Struct Mol Biol 20: 908-913, 2013.

36. Cheetham SW, Gruhl F, Mattick JS and Dinger ME: Long noncoding RNAs and the genetics of cancer. Br J Cancer 108: 2419-2425, 2013

37. Betel D, Wilson M, Gabow A, Marks DS and Sander C: The microRNA.org resource: Targets and expression. Nucleic Acids Res 36: D149-D153, 2008.

38. Peterson JM, Wei Z and Wong GW: CTRP8 and CTRP9B are novel proteins that hetero-oligomerize with C1q/TNF family members. Biochem Biophys Res Commun 388: 360-365, 2009.

39. Chen Y and Su Z: Reveal genes functionally associated with ACADS by a network study. Gene 569: 294-302, 2015.

40. Bartel DP: MicroRNAs: Target recognition and regulatory functions. Cell 136: 215-233, 2009. 